\title{
Does Psa affect kiwifruit susceptibility to leafrollers?
}

\author{
M.G. Hill \\ The New Zealand Institute for Plant E Food Research Limited, 412 No 1 Rd Te Puke, 3182, \\ New Zealand \\ Corresponding author: garry.hill@plantandfood.co.nz
}

\begin{abstract}
Analysis of kiwifruit industry pest monitoring and spray diary data showed increases in the incidence of leafroller larvae on fruit during crop monitoring in the 2011/12 season in regions with the virulent strain of the disease Pseudomonas syringae pv. actinidiae (Psa-V). Four factors were considered as explanations: Psa-V infection and its effects on plant defences, leafroller sprays, Psa-V sprays and leafroller sampling. The most plausible cause of the increased leafroller incidence is Psa-V infection. A putative mechanism is hormonal 'cross-talk' resulting in a trade-off between pest and pathogen resistance. The susceptibility of vines to leafrollers in Psa-V-infected regions appears to persist for several weeks longer after fruit set than in uninfected vines. The paper discusses the need to develop a better understanding of the interactions of biotic and abiotic stressors on plant defence responses to pests and diseases and to incorporate these into a plant-defence-centred approach to Integrated Pest Management.
\end{abstract}

Keywords kiwifruit, Psa, leafrollers, plant resistance, IPM, cross-talk.

\section{INTRODUCTION}

The Zespri pest and disease management decision support system KiwiGreen ${ }^{\circledR}$ has been used since 1997 for ensuring that pesticides are applied only according to demonstrated need. According to KiwiGreen ${ }^{\circledR}$ protocols, a certain number of spray applications are allowed on 'Hayward' kiwifruit (Actinidia deliciosa) crops pre-flowering and in the first 6 weeks after flowering. After this time, from approximately January onwards, sprays can be applied for leafroller control only following crop monitoring, when the proportion of infested fruit bunches exceeds the threshold of $0.5 \%$. Since 2007 a selection of the spray diary and pest monitoring records have been stored in electronic format and these data are being used to investigate the interaction between pest management practices (e.g. chemicals used, spraying practices) and pest monitoring outcomes (decisions to spray the crop against leafroller or scale insect attack) (M.G. Hill, unpublished data).

The proportion of first-of-the-season (i.e. early summer) leafroller monitoring events that were above the spray threshold in conventionally grown 'Hayward' kiwifruit (hereafter 'leafroller incidence') increased from 15\% (1216/8277 blocks) in the 2011 season (the year the crop was harvested) to $42 \%$ (2430/5801 blocks) in 2012. The figures for the four previous seasons were: 2007, 21\% ( $\mathrm{n}=2910) ; 2008,29.8 \%$ (4441); 2009, $34.4 \%$ (7853); and 2010, 20.1\% (8277). Clearly, leafroller incidence on fruit changes somewhat from year to year, presumably based upon a range of factors relating to the environment and management practices. However, the change from 2011 to 2012 was unprecedented, and coincided 
with the appearance and spread of the 'virulent' strain of the bacterial disease Pseudomonas syringae pathovar actinidiae (Psa-V). Psa-V was discovered in Te Puke in November 2010 and spread rapidly throughout Bay of Plenty and to other regions (www.kvh.org.nz). Thus, a leading hypothesis to explain these results is that the disease has altered the susceptibility of the vines to leafroller attack. This hypothesis is based upon an understanding of plant defence systems. Pseudomonas syringae is known to stimulate plants to activate the salicylic acid (SA) hormonal defence pathway (Pieterse et al. 2012). This response has been shown to lead to a down-regulation of the jasmonic acid (JA) signalling pathway (Glazebrook 2005; Zarate et al. 2007; Robert-Seilaniantz et al. 2011; Thaler et al. 2012), which is the main hormonal pathway used by plants to protect against attack from chewing insects (Erb et al. 2012). However, there are other possible explanations.

This paper tests four hypotheses to explain these results: (1) infection by Psa-V leads to a reduction in defences to plant-chewing insects; (2) fewer leafroller control sprays are applied in areas with Psa-V compared with areas without Psa-V; (3) sprays applied to combat Psa-V affect susceptibility of the plant to leafrollers; and (4) changes in the dates of monitoring between years is responsible for the observed changes in leafroller incidence because plant susceptibility to leafroller incidence or susceptibility declines over time from midsummer onwards (in days after fruit set).

\section{MATERIALS AND METHODS}

The proportion of orchards with positive Psa-V tests in April 2011, November 2011 and April 2012 was obtained from Kiwifruit Vine Health (http://www.kvh.org.nz/). The analyses undertaken to test the four hypotheses outlined above are described below.

\section{Testing the effects of Psa-V infection}

Using the pest monitoring records from 2007 to 2012, leafroller incidence (the proportion of first-of-the-season (i.e. early summer) leafroller monitoring events that were above the spray threshold in conventionally grown
'Hayward' fruit) was calculated for each supply region. Supply regions that were known to have Psa-V at the start of the 2011-12 season (Psa+ = Tauranga, Te Puke, Pukehina, Maketu and Whakatane) were compared with those that were Psa-V-free at the start of that season (Psa$=$ Franklin, Auckland, Whangarei and Kerikeri). Leafroller incidence $( \pm 95 \%$ confidence intervals (Agresti \& Coull 1998)) was calculated for all orchards over the whole season and separately for orchards monitored within each 2-week period after fruit set.

\section{Leafroller sprays}

The mean number of spray applications against leafrollers in 2012 was calculated and compared between regions with and without Psa-V.

\section{Psa-V sprays}

The mean number of spray applications against Psa-V was calculated for regions with and without Psa-V in 2012. The sprays were divided into categories of (1) all sprays against Psa-V, (2) copper sprays (the majority of protectants are copper-based) and (3) acibenzolar-S-methyl. This latter chemical is claimed to act as an 'elicitor', by mimicking the defence phytohormone salicylic acid. The other sprays are thought to act primarily as protectants.

\section{Monitoring dates}

Because leafroller incidence declines over time (in days after fruit set - see results section and McKenna \& Stevens 2007), the average date on which pest monitoring took place should affect estimates of leafroller incidence, irrespective of other factors. The date of the first monitoring event in summer is usually 5-7 weeks after fruit set but it is decided by the grower or pest monitoring centre and varies substantially. The mean date of monitoring was calculated for blocks in regions with and without Psa-V for the 6 years of monitoring data.

From the same data set, mean leafroller incidence was calculated at 2-week intervals after fruit set for each year from 2007 to 2012 to show the relationship of leafroller incidence to time in 2-week intervals, after fruit set. 


\section{RESULTS}

The percentage of orchards with Psa-V in April 2011 in Psa+ regions was 28\% $(\mathrm{n}=683)$ and $0 \%$ $(\mathrm{n}=33)$ in Psa- regions. Psa-V was discovered on 3 orchards in Franklin region (previously Psa-) on 28 Nov 2011. In December 2011 the percentage of orchards confirmed to have Psa infection in the Psa+ regions was $46 \%(n=1885)$ and in Psa- regions, $1 \%(n=323)$. By April 2012, these figures were Psa+ 58\% and Psa- 2\% (http://www. kvh.org.nz). The five Psa-V-infected orchards in the Psa- region were included in the analysis but are considered to have caused negligible bias to the results.

\section{Possible effects of Psa-V on kiwifruit resistance}

The annual pattern of leafroller incidence in conventionally grown A. deliciosa 'Hayward' between 2007 and 2011 was similar in the two regional groups, but leafroller incidence was substantially and consistently lower in the Psa+ regions than in the Psa- regions (Figure 1). In the
2011/12 growing season, the leafroller incidence increased significantly at Psa + sites to a rate above that in the Psa- sites (Figure 1). This was consistent in all seven Psa+ regions (Figure 2), but the increase in some regions was much higher than others. By contrast, leafroller incidence declined or remained the same in all four Psa- regions. Leafroller incidence in 2-week intervals after fruit set showed a marked decline beginning 4-6 weeks after fruit set in each year except during the 2012 season in the Psa+ regions (Figure 3).

\section{Leafroller spray applications}

The hypothesis that the difference in leafroller incidence was due to fewer pre-monitoring leafroller control sprays applied in Psa+ than Psaregions is not supported by the spray data (Table 1 ).

\section{Psa-V sprays}

On average twice as many Psa-protectant and elicitor sprays were applied in the Psa+ regions than in the Psa- regions (Table 1). However,

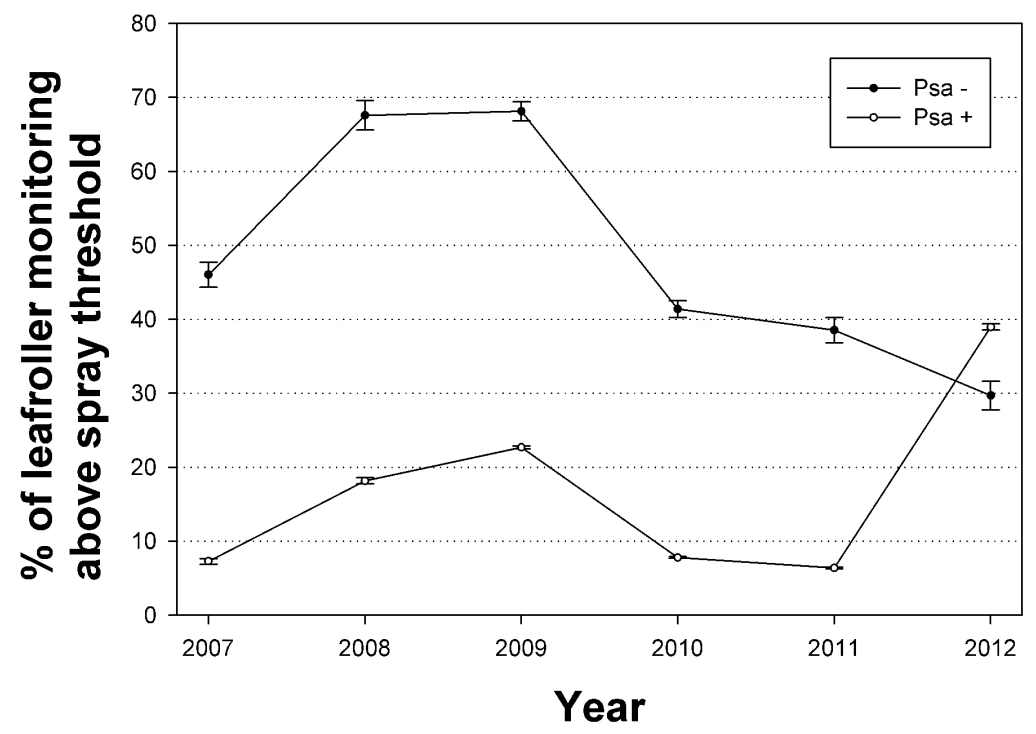

Figure 1 Leafroller incidence based on proportion of monitoring events above the spray threshold on 'Hayward' kiwifruit between 2007 and 2012 in regions without Pseudomonas syringae pv. actinidiae (Psa) in the 2011/12 season (Franklin, Auckland, Whangarei and Kerikeri) and those with Psa (Tauranga, Te Puke, Maketu, Paengaroa and Whakatane). Vertical bars are 95\% confidence intervals (CIs) (mean sample size $=1941$ (range 675 to 4691$)$ ). In some cases CIs are too small to be accurately represented. 


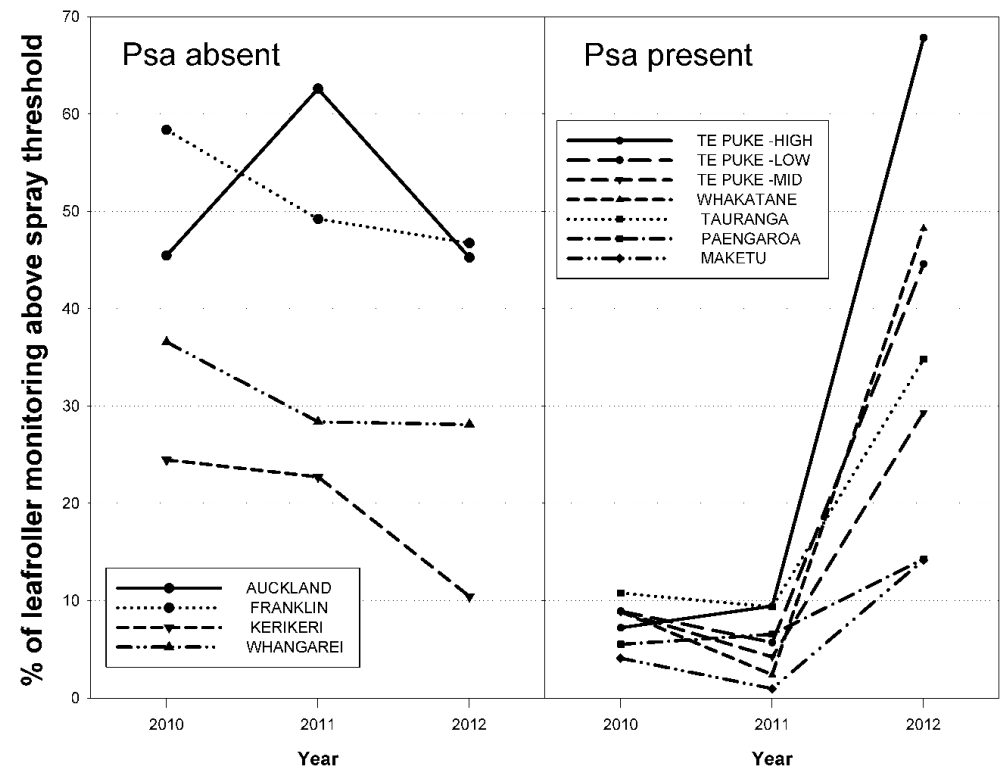

Figure 2 Leafroller incidence on 'Hayward' kiwifruit between 2010 and 2012 for four regions without Pseudomonas syringae pv. actinidiae (Psa) and seven regions with Psa in the 2011/12 season.

substantial amounts of sprays were applied prophylactically in the Psa- regions and more acibenzolar-S-methyl sprays (a salicylic acid mimic) were applied in the Psa- regions than in the Psa+ regions (Table 1).

\section{Timing of monitoring}

Mean days to the first monitoring event (in days after fruit set) in 2012 for blocks above or below the spray threshold in the Psa+ regions were respectively 2.5 and 3.6 days earlier than the average for the previous 5 years, while the dates for the Psaregions were 3.5 earlier and 4.7 days later than the average for the previous 5 years (Table 2). Thus, the variation in monitoring dates in 2012 compared with 2011 may have contributed up to 7 days ( $~ 5$ days because of late sampling in the Psa- region and $\sim 2$ days for early sampling in the Psa+ region) to the observed difference in leafroller incidence between the Psa+ and Psa- regions in 2012. Based upon the relationship between leafroller incidence and days after fruit set in Figure 3 from 6-10 weeks after fruit set, leafroller incidence declined at this time on average by approximately $1.3-$ $1.4 \%$ per day in the Psa- region and by $0.6-0.7 \%$ per day in the Psa+ regions (Figure 3). Together, these data suggest that compared with average sampling dates for the previous 5 years, in 2012 the observed proportion of monitoring events over the threshold may have been $\sim 7-8 \%$ higher than expected for the Psa+ regions and 1-2\% lower than expected for the Psa- regions as a result of the difference in monitoring dates and the relationship between monitoring date and leafroller incidence.

\section{DISCUSSION}

The results of this analysis are consistent with a hypothesis that Psa-V infection of 'Hayward' kiwifruit increases its susceptibility to leafrollers. The vines appear to remain susceptible to leafroller attack for a longer period of time after fruit set than do uninfected vines (Figure 3).

The effect of the large number of Psa-V sprays applied in the Psa+ regions (mean 6.6) on leafroller incidence cannot be determined from this analysis and it is possible that they may also be influencing vine susceptibility. However, a significant number of sprays to combat Psa-V were applied prophylactically in the Psa- regions (mean 3.4), and more acibenzolar-S-methyl, a powerful salicylic 
Table 1 Mean numbers of sprays and spray combinations per block applied to 'Hayward' kiwifruit in regions with (Psa+) and without (Psa-) Pseudomonas syringae pv. actinidiae in 2012 (data from Zespri spray diaries). 'total Psa sprays' includes 23 different active ingredients including copper compounds, elicitors, oxidising agents and microbial products. Seven leafroller-toxic active ingredients were applied.

\begin{tabular}{lcc}
\hline & Psa- & Psa+ \\
\hline Total Psa sprays & 3.4 & 6.6 \\
Copper sprays & 1.6 & 3.9 \\
Acibenzolar-S-methyl $^{1}$ & 0.9 & 0.5 \\
Pre-monitoring leafroller sprays $^{1}$ & 1.4 & 2.2 \\
\hline
\end{tabular}

${ }^{1}$ Salicylic acid mimic and plant defence elicitor.

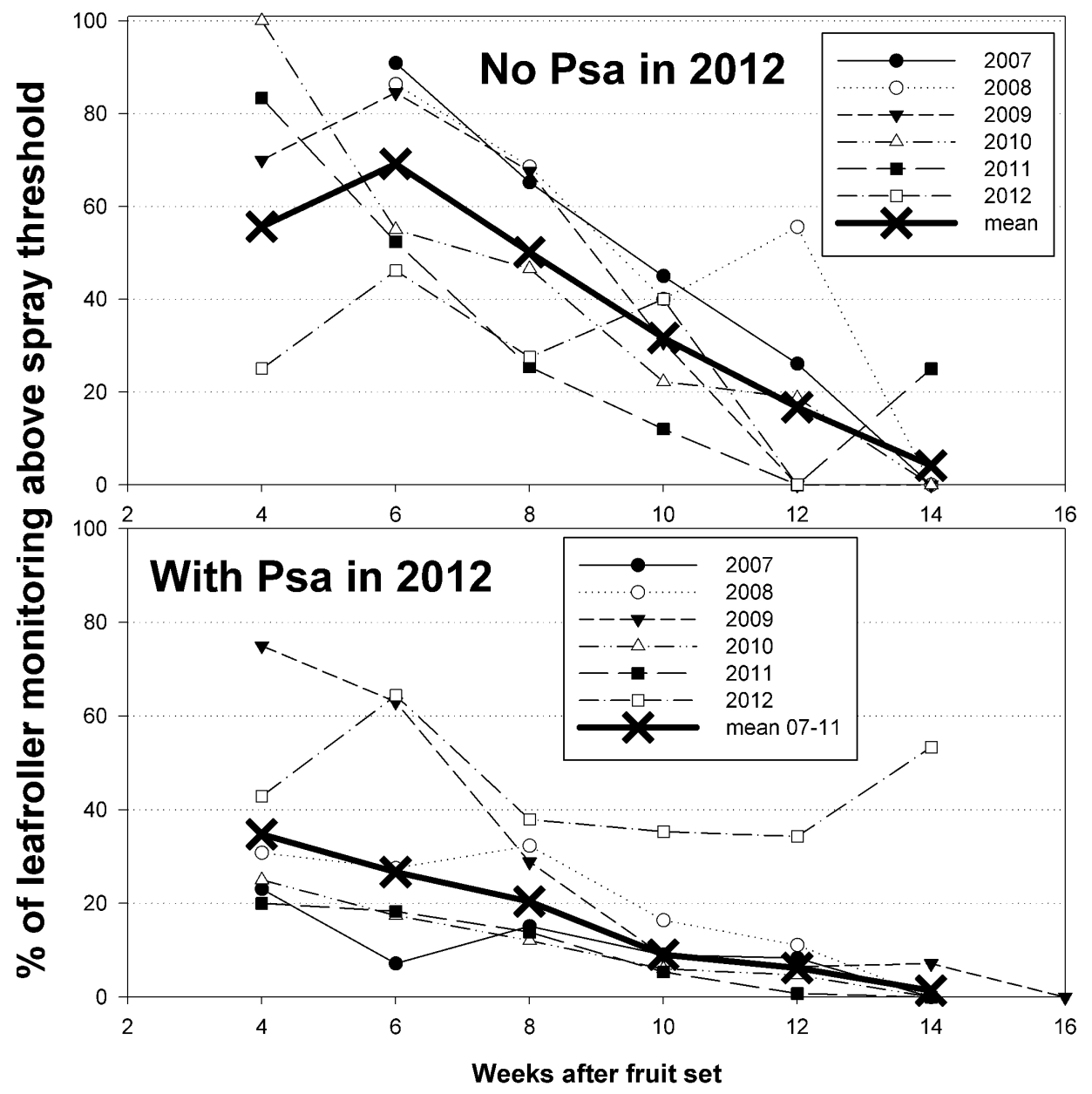

Figure 3 Leafroller incidence on 'Hayward' kiwifruit at 2-week intervals after flowering in sites with and without Pseudomonas syringae pv. actinidiae (Psa) in 2012 for the 6 years from 2007 to 2012. 
Table 2 Mean number of days after 'Hayward' kiwifruit fruit set until the first leafroller monitoring event for blocks that were above the spay threshold ('Spray') or below the spray threshold ('NoSpray') in regions with (Psa+) and without (Psa-) Pseudomonas syringae pv. actinidiae (Psa-V) in 2012. All = NoSpray + Spray blocks combined (data from Zespri spray diaries).

\begin{tabular}{lccccccc}
\hline & \multicolumn{3}{c}{ Psa- } & & \multicolumn{3}{c}{ Psa +} \\
\cline { 2 - 5 } \cline { 7 - 8 } Year & NoSpray & Spray & All & & NoSpray & Spray & All \\
\hline 2007 & 59.8 & 39.3 & 50.3 & & 56.6 & 55.0 & 56.5 \\
2008 & 54.8 & 48.1 & 50.3 & & 54.0 & 49.3 & 53.2 \\
2009 & 51.1 & 42.2 & 45.0 & & 59.4 & 45.4 & 56.2 \\
2010 & 41.3 & 40.7 & 41.0 & & 55.5 & 49.4 & 55.1 \\
2011 & 55.6 & 38.2 & 49.3 & & 57.5 & 50.5 & 57.0 \\
2012 & 49.0 & 46.4 & 48.2 & & 53.0 & 47.4 & 51.3 \\
\hline Mean 07-11 & 52.5 & 41.7 & 47.2 & & 56.6 & 49.9 & 55.6 \\
SD 07-11 & 7.0 & 3.9 & 4.1 & & 2.0 & 3.4 & 1.5 \\
\hline
\end{tabular}

acid mimic, was applied in the Psa- regions than in the Psa+ regions. Thus it seems unlikely that these sprays were a major cause of the increased leafroller incidence in the Psa+ regions.

There would appear to be a small but probably significant bias in estimates of leafroller incidence due to variations in sampling times between years. However, there is a possibility that the response of the vines to Psa-V in 2012 may have affected that relationship, making the vines more susceptible to leafroller attack for longer (Figure 3), and thereby reducing the implied monitoring date bias. Whether monitoring dates have introduced a significant bias or not, the size of the effect cannot explain the results obtained in 2012 in the Psa+ regions.

Analyses of industry-derived data sets such as the pest monitoring and spray diary data used here must be treated with caution because of possible biases in data collected. But the value of data sets such as these lie in their ability to reveal interactions occurring on a large scale, under field conditions and with large sample sizes. Use of these data sets has revealed a potential link between Psa-V and vine susceptibility to leafrollers and it has shown that there may be an inherent difference in pest incidence between regions. These hypotheses are testable under controlled conditions.

Hypotheses to explain the inherent difference in leafroller incidence between the regions might include ecological factors, such as landscape effects and natural enemy incidence. But it is also possible that the differences in leafroller pest incidence are related to inherent vine susceptibility resulting from abioticstresses (e.g.soil water stress, weather-related factors) (Cao et al. 2011; Lee \& Luan 2012; Walters et al. 2013). Soils in the Auckland and Franklin regions (typically granular and brown soil orders; http://soilsdev.waikatoregion.govt.nz/) are more likely to lead to shallow rooting and increased water stress in vines during summer than Bay of Plenty volcanic (pumice) soils. This soil-related plant stress hypothesis to explain pest incidence is testable experimentally, whereas ecological explanations are very difficult to test.

Recent advances in knowledge of plant defence systems has shown that a small number of hormonal pathways control a range of complex and inter-related interactions between plant responses to pathogen and pest attack and other biotic stressors (Erb et al. 2012; Pieterse et al. 2012; Walters et al. 2013). These pathways also respond to a wide range of abiotic stress factors (Erb et al. 2011; Walters et al. 2013), which may result in defence pathway antagonism (Robert-Seilaniantz et al. 2011; Atkinson \& Urwin 2012). The two main plant defence pathways, jasmonic acid (JA) and salicylic acid (SA), control responses to different groups of pests and diseases. Plants defend themselves against biotrophic or hemibiotrophic pathogens such as $\mathrm{Psa}-\mathrm{V}$ principally by stimulating the SA pathway (Louis et al. 
2012), while JA is the main defence pathway for necrotrophic pathogens and leaf chewing insects (Erb et al. 2012; Thaler et al. 2012). These two pathways have been shown to date to be largely antagonistic and this so-called 'cross-talk' can result in the plant shutting down the response of one pathway while increasing signalling in the other during the plant's defensive response to a specific pathogen or insect (Robert-Seilaniantz et al. 2011).

Pathogens and some insects are known to produce chemical elicitors that mimic chemicals in the plant's hormonal defence pathways, causing them to be turned on or off (Howe \& Jander 2008; Bonaventure et al. 2011; Sarmento et al. 2011a, b). Synthetic homologues of chemicals in these defence pathways (e.g. acibenzolar-S-methyl (ASM), a SA mimic) are registered for spraying onto crops to stimulate the plant's immune system (Young 2013). A recent laboratory study has shown that a spray application of ASM to potted kiwifruit plants can cause a decrease in Psa-V symptoms (J.L. Vanneste, Plant \& Food Research, unpublished data), and a field trial in Italy has shown that ASM can increase concentrations of SA and reduce Psa-V incidence and plant growth in commercially grown A. chinensis 'Hort16A' vines (Kay et al. 2012). Elicitors are becoming standard tools in the fight against Psa-V and other biotrophic and hemibiotrophic pathogens (Walters et al. 2013), and these chemicals are being hailed as key components of future pathogen 'integrated' management systems using, for example, a combination of elicitors and fungicides (Walters et al. 2013). This development is likely to have an increasingly important effect on insect pest management and Integrated Pest Management (IPM) systems in the future.

In developing future IPM systems, it would seem timely to consider taking a plant-defencecentred perspective, acknowledging that when the plant reacts to one stressor it may be making itself more or less resistant to attack from another stressor. Knowledge of plant physiological responses to a range of biotic and abiotic stressors should also assist plant breeders to develop optimal plant resistance genotypes in future varieties. Research to develop ways of optimising plant defence responses to a suite of factors is in its infancy, but rapid advances in genomics, and research on plant defence responses to interacting biotic and abiotic factors (e.g. Pineda et al. 2012; Soler et al. 2012), should enable this degree of complexity to be incorporated into future IPM systems.

\section{ACKNOWLEDGEMENTS}

Zespri International Ltd supplied the pest monitoring and spray diary data. Kiwifruit Vine Health provided data on Psa incidence. David Logan, Tony Reglinski and a reviewer provided valuable comments on a draft.

\section{REFERENCES}

Agresti A, Coull BA 1998. Approximate is better than exact for interval estimation of binomial proportions. The American Statistician 52: 119-126.

Atkinson NJ, Urwin PE 2012. The interaction of plant biotic and abiotic stresses: from genes to the field. Journal of Experimental Botany 63: 3523-3543.

Bonaventure G, VanDoorn A, Baldwin IT 2011. Herbivore-associated elicitors: FAC signaling and metabolism. Trends in Plant Science 16: 294-299.

Cao FY, Yoshioka K, Desveaux D 2011. The roles of ABA in plant-pathogen interactions. Journal of Plant Research 124: 489-499.

Erb M, Meldau S, Howe GA 2012. Role of phytohormones in insect-specific plant reactions. Trends in Plant Science 17: 250-259.

Erb M, Koellner TG, Degenhardt J, Zwahlen C, Hibbard BE, Turlings TCJ 2011. The role of abscisic acid and water stress in root herbivore-induced leaf resistance. New Phytologist 189: 308-320.

Glazebrook J 2005. Contrasting mechanisms of defense against biotrophic and necrotrophic pathogens. Annual Review Phytopathology 43: 205-227.

Howe GA, Jander G 2008. Plant immunity to insect herbivores. Annual Review of Plant Biology 59: 41-66. 
Kay C, Onorato R, Gentili A 2012. Focus on elicitors. Actigard ${ }^{\mathrm{TM}}$ root drenching, residues and growth impact on Hort16A. Kiwifruit Journal, November/Decenber: 53-58.

Lee SC, Luan S 2012. ABA signal transduction at the crossroad of biotic and abiotic stress responses. Plant Cell and Environment 35: 53-60.

Louis J, Gobbato E, Mondal HA, Feys BJ, Parker JE, Shah J 2012. Discrimination of arabidopsis PAD4 activities in defense against green peach aphid and pathogens. Plant Physiology 158: 1860-1872.

McKenna CM, Stevens PS 2007. A comparison of lepidopteran damage to 'Hort16A' and 'Hayward' kiwifruit. New Zealand Plant Protection 60: 254-258.

Pieterse CMJ, Van der Does D, Zamioudis C, Leon-Reyes A, Van Wees SCM 2012. Hormonal modulation of plant immunity. In: Schekman R, Goldstein L, Lehmann R ed. Annual Review of Cell and Developmental Biology 28: 489-521.

Pineda A, Zheng SJ, Loon JJAv, Dicke M 2012. Rhizobacteria modify plant-aphid interactions: a case of induced systemic susceptibility. Plant Biology 14: 83-90.

Robert-Seilaniantz A, Grant M, Jones JDG 2011. Hormone crosstalk in plant disease and defense: more than just JASMONATESALICYLATE antagonism. In: VanAlfen NK, Bruening G, Leach JE ed. Annual Review of Phytopathology 49: 317-343.
Sarmento RA, Lemos F, Dias CR, Kikuchi WT, Rodrigues JC, Pallini A, Sabelis MW, Janssen A 2011a. A herbivorous mite down-regulates plant defence and produces web to exclude competitors. Plos One 6: e23757.

Sarmento RA, Lemos F, Bleeker PM, Schuurink RC, Pallini A, Oliveira MG, Lima ER, Kant M, Sabelis MW, Janssen A 2011b. A herbivore that manipulates plant defence. Ecology Letters 14: 229-236.

Soler R, Badenes-Perez FR, Broekgaarden C, Zheng S, David A, Boland W, Dicke M 2012. Plant-mediated facilitation between a leaffeeding and a phloem-feeding insect in a brassicaceous plant: from insect performance to gene transcription. Functional Ecology 26: 156-166.

Thaler JS, Humphrey PT, Whiteman NK 2012. Evolution of jasmonate and salicylate signal crosstalk. Trends in Plant Science 17: 260-270.

Walters DR, Ratsep J, Havis ND 2013. Controlling crop diseases using induced resistance: challenges for the future. Journal of Experimental Botany 64: 1263-1280.

Young S ed. 2013. New Zealand Novachem Agrichemical Manual. Agrimedia Ltd, Christchurch. 768 p.

Zarate SI, Kempema LA, Walling LL 2007. Silverleaf whitefly induces salicylic acid defenses and suppresses effectual jasmonic acid defenses. Plant Physiology 143: 866-875. 